\title{
Correction to: Surface elastic-based nonlinear bending analysis of functionally graded nanoplates with variable thickness
}

\author{
Saeid Sahmani ${ }^{1}$, Babak Safaei ${ }^{2}$, Fadi Aldakheel ${ }^{3, \text { a }}$ \\ ${ }^{1}$ School of Science and Technology, The University of Georgia, 0171 Tbilisi, Georgia \\ 2 Department of Mechanical Engineering, Eastern Mediterranean University, North Cyprus via Mersin 10, \\ Famagusta, Turkey \\ ${ }^{3}$ Institute of Continuum Mechanics, Leibniz Universitaet Hannover, 30823 Garbsen, Germany
}

(C) The Author(s), under exclusive licence to Società Italiana di Fisica and Springer-Verlag GmbH Germany, part of Springer Nature 2021

Correction to: Eur. Phys. J. Plus (2021) 136:676

$$
\text { https://doi.org/10.1140/epjp/s13360-021-01667-7 }
$$

Equation 6a:

$$
\mathbb{F}(z)=\sin (\pi z / h)-z
$$

The original version of this paper was inadvertently published with an incorrect formula. The correct formula is:

$$
\mathbb{F}(z)=(h / \pi) \sin (\pi z / h)-z
$$

The original article has been corrected. We apologise for any inconvenience caused to our readers.

The original article can be found online at https://oi.org/10.1140/epjp/s13360-021-01667-7.

\footnotetext{
a e-mail: aldakheel@ikm.uni-hannover.de (corresponding author)
} 